\title{
Association study of GFAP Polymorphisms with schizophrenia in Han Chinese population
}

\author{
Wenjuan Yu \\ Shanghai Mental Health Center \\ sidi he \\ Shanghai Mental Health Center \\ Yimin $\mathrm{Yu}$ \\ Shanghai Mental Health Center \\ Shen $\mathrm{He}$ \\ Shanghai Mental Health Center \\ Qingqing Xu \\ Shanghai Mental Health Center \\ Shunying Yu \\ Shanghai Mental Health Center \\ Jingjing Huang \\ Shanghai Mental Health Center \\ huafang Li ( $\nabla$ smhcmict@163.com ) \\ Shanghai Mental Health Center https://orcid.org/0000-0003-2384-8998
}

\section{Research article}

Keywords: Glial fibrillary acidic protein, schizophrenia, SNP, haplotype, eQTL

Posted Date: January 23rd, 2020

DOI: https://doi.org/10.21203/rs.2.21670/v1

License: (c) (i) This work is licensed under a Creative Commons Attribution 4.0 International License. Read Full License 


\section{Abstract}

\section{INTRODUCTION}

Glial fibrillary acidic protein (GFAP) is considered to be an astrocyte activation marker. Alterations in structural and functional molecules expressed by astrocytes may play a role in the pathophysiology of schizophrenia (SCZ). In the present study, the single nucleotide polymorphisms (SNPs) of GFAP were analyzed for association with schizophrenia in Han population.

\section{METHODS}

Three tag SNPs within GFAP were genotyped in 629 SCZ patients and 655 healthy controls in the Han Chinese population.The schizophrenia database(SCZB) was used to investigate whether GFAP is differentially expressed in the brain regions of schizophrenia and healthy control individuals.In addition, expression quantitative trait loci(eQTL) analysis was used to investigate differential GFAP expression between different genotypes.

\section{RESULTS}

It was shown that the rs3785891 GFAP polymorphism may be associated with schizophrenia $(p=0.04)$. There were no significant differences in the expression of hippocampal, prefrontal cortex or cortical GFAP between schizophrenia patients and control individuals. Furthermore,the brain eQTL analysis revealed a significant association between the rs3785891 polymorphism and GFAP expression in the intralobular white matter $(p=0.04)$, thalamus $(p=0.024)$, hippocampus $(p=0.011)$, and occipital cortex $(p=0.0075)$.

\section{CONCLUSIONS}

This study shows that rs3785891 may be linked to schizophrenia susceptibility in the Han population. Further study is required for clarification the role of genetic variation around these SNPs in expression pattern of the GFAP gene, which may be involved in schizophrenia pathogenesis.

\section{Introduction}

Schizophrenia (SCZ) is a serious life-long psychiatric disorders affecting approximately $1 \%$ of the population worldwide. It is a heterogeneous ,complex behavioral and cognitive syndrome.Its average heritability is estimated at $81 \%$ with a range of $73 \%-90 \%[1]$. In the past few decades, several studies have been conducted on schizophrenia susceptibility genes. However, the exact genetics and pathogenesis of SCZ remain unclear.

Glial fibrillary acidic protein (GFAP) is a type III intermediate filamentous protein that exists in monomeric form and is expressed almost exclusively in astrocytes. GFAP is involved in glial plasticity and neuroinflammatory processes in response to emotional experiences ${ }^{[2]}$. The expression of GFAP is regulated by many factors, including cytokines and growth factors ${ }^{[3]}$ and is considered to be a marker of astrocyte activation ${ }^{[4]}$. Astrocytes are the most abundant and diverse cells in the central nervous system ${ }^{[5]}$ and are involved in many important regulatory functions that maintain brain homeostasis. Astrocytes can form a triple synaptic structure with specialized presynaptic and postsynaptic regions of neurons and play an important role in integrating and processing complex cognitive tasks ${ }^{[6]}$. They have recently been recognized as active participants in dendritic spine elimination, synaptic development via the induction of synapse formation and neurotransmission regulation, as well as plasticity. Dendritic spine and synaptic damage are characteristic of many neurological disorders, including autism spectrum disorders, SCZ and Alzheimer's disease $\mathrm{e}^{[7]}$.

With the improved understanding of astrocyte function within synapses, the role of astrocytes in SCZ has received increased attention.Williams et al (2013) found that,compared to the control group, a significant decrease in astrocyte density was observed in SCZ relative to normal controls in the cingulate grey matter, cingulate white matter and the midline of the corpus callosum ${ }^{[8]}$. This suggests that there is a specific glial cell type change in SCZ. A recent animal study found a significant increase of GFAP in the hippocampus of Gunn rats with schizophrenic-like behavior ${ }^{[9]}$. Sun et al (2017) first observed a significant increase in GFAP in models involving socially isolated animals $^{[10]}$. Social isolation is thought to be a cause of SCZ spectrum disorders.

Given that GFAP may be involved in the etiological and therapeutic mechanisms of SCZ, this study investigated whether genetic common variants in the GFAP gene were associated with the risk of SCZ.

\section{Materials And Methods}


The case-control samples were included 629 patients with SCZ ( $53.7 \%$ men and $46.3 \%$ women; mean age:39.85 \pm 14.85 years) and 655 healthy controls (49.8\% men and 50.2\% women; mean age: $35.14 \pm 17.02$ years). SCZ were recruited in three wards of Shanghai Mental Health Center wards from 2011 to 2016 .The inclusion criteria were as follows:1) patients must have met the diagnostic criteria for SCZ according to two experienced senior psychiatrists, as per the Diagnostic and Statistical Manual of Mental Disorders, Fourth Edition (DSMIV),2)no serious physical illness, including a history of cancer or stroke(s), must be present, and they must consent to take part in this study. The healthy control group comprised volunteers recruited by the Shanghai Community and the Shanghai Mental Health Center. In a brief interview with a senior psychiatrist, volunteers had neither serious physical illness, mental illness nor a family history of mental health conditions. All participants are Han Chinese. This study was approved by the Ethics Committee of the Shanghai Mental Health Center. Written consent was obtained from all subjects or their guardians.

\subsection{SNP selection and genotyping}

The human GFAP gene, located on chromosome 17q21 (44905626..44915552) (GRCh38.p12 (GCF 000001405.38)), includes nine exons and eight introns ${ }^{[13]}$. First, tag SNPs were selected to cover the whole gene as presented in the 1000 Genomes Project

(http://www.1000genomes.org/). Tag SNPs required an $r^{2} \geq 0.8$, and a minor allele frequency (MAF) $\geq 0.05$ in the Han Chinese population of Beijing (HCB). As shown in Fig. 1, five tag SNPs, rs3785891 (exon4), rs2289681 (exon5), rs8067029 (exon5), rs2289671 (exon5), rs3744469 (exon4), were selected. Of these, rs8067029 and rs2289671 were not detected at the time of screening, so results are representative of rs3785891, rs2289681 and rs3744469 only.

Genomic DNA was extracted from peripheral blood using the Tiangen DNA isolation kit according to standard laboratory procedures (Blood genomic DNA extraction kit, Tiangen Biotech Inc. Beijing, China). The three SNPs were classified using the Taqman (Applied Biosystems, Foster City, CA USA) genotyping method. The PCR amplification reaction volume of $5 \mathrm{uL}$ consisted of $2.46 \mu \mathrm{L}$ master mix, $2.5 \mu \mathrm{L}$ DNA, and $0.04 \mu \mathrm{L}$ Taqman probe. PCR amplification was initiated at $95^{\circ} \mathrm{C}$ for $10 \mathrm{~min}$, then 45 cycles at $92^{\circ} \mathrm{C}$ for 15 seconds, and $60{ }^{\circ} \mathrm{C}$ for $1 \mathrm{~min}$. Following amplification, allele-specific fluorescence was measured on an ABI PRISM 7900 sequencer (Applied Biosystems).

For quality control, the case and control status was blinded during all genotyping processes. Simultaneously, $5 \%$ of the samples were repeated and the results were $100 \%$ consistent.

\subsection{Brain eQTL Analysis for SNPs Associated with SCZ Risk}

In order to further explore the differences between GFAP expression in various brain regions of individuals with different genotypes, expression quantitative trait loci(eQTL) analysis was performed using the brain eQTL database. This is a large exon-specific eQTL dataset covering 10 human brain regions [inferior olivary nucleus (MEDU), intralobular white matter (WHMT), substantia nigra (SNIG), thalamus (THAL), hippocampus (HIPP), putamen (PUTM), frontal cortex (FCTX), occipital cortex (OCTX), temporal cortex (TCTX), and cerebellar cortex (CRBL)]. More detailed information about this database can be found in the original literature ${ }^{[14]}$.

\subsection{Analysis of brain GFAP expression}

It is well known that SCZ originates from abnormal brain structure and function ${ }^{[11]}$, and that the dysregulation of gene expression plays an important role in the pathogenesis of the disease. This study used the schizophrenia database (SZDB) (http://www.szdb.org/), as a newly developed comprehensive resource for SCZ research ${ }^{[12]}$, to detect whether there were differences in GFAP expression in brain regions between SCZ patients and healthy controls.

\subsection{Statistical analysis}

The statistical power of the case-control sample size was calculated using QUANTO software (version 1.2.4.). The prevalence of SCZ was hypothesized to be $1 \%$, and the moderate odds ratio $(\mathrm{OR})$ at 1.3. The inheritance model was set as an additive model with the sample size of case-control pairs set to 700. As the MAF of the selected SNPs ranged from $2.0-26.0 \%$ (Table1), the power of the samples ranged from $16.6-$ $87.8 \%$.

The SHEsis online software ${ }^{[15]}$ (http://analysis2.bio-x. cn/myAnalysis.php) was used to test the Hardy Weinberg equilibrium (HWE), while the chi-square test was used to compare the allele and genotype frequencies of the SCZ and control group. All statistical tests were two-sided, and $p<0.05$ was considered statistically significant. 
The genotyping data of this study were used to construct linkage disequilibrium (LD) blocks using Haploview (version 4.1) to further analyze the relationship between different haplotypes and the risk of SCZ.Frequencies below $0.05 \%$ were considered to be rare and were excluded from further analysis. The permutation procedure was executed for multiple comparisons, and $p<0.05$ was considered significant.

\section{Results}

\subsection{Brain GFAP expression}

Brain GFAP expression data between the SCZ and healthy control groups were extracted from the SZDB. Figure 2 shows that there were no significant differences in GFAP expression in the hippocampus, prefrontal cortex or stratum between the SCZ and control subjects (corrected $P=0.82,0.444$, and 0.566 , respectively).

\subsection{Allele and genotype analysis}

All SNPs were in HWE with call rates for each SNP above $98.3 \%$ in the two groups. Analysis of all three SNPs revealed a significant difference in genotype distribution between case and control individuals for rs3785891 $(p<0.05$, Table 1$)$.

\subsection{Linkage disequilibrium}

Using Haploview software (version 4.1), LD blocks were built and are shown in Fig. $3^{[16]}$. One block was constructed for rs2289681 and rs3785891. Table 2 shows the results of haplotype frequencies between SCZ patients and healthy controls. There was no significant correlation between the different haplotypes and the risk of SCZ ( $p>0.05)$.

\subsection{Brain eQTL analysis}

To explore the role of various polymorphisms in the brain GFAP expression, eQTL analysis was performed. Results showed a significant association between the rs3785891 polymorphism and GFAP expression in the WHMT $(p=0.04)$, THAL $(p=0.024), \operatorname{HIPP}(p=0.011)$, and OCTX $(p=0.0075)$ regions, while there was a clear trend towards significance in the FCTX $(p=0.053)$ region as well. These results emphasize that the expression of the TT genotype was higher than that of the T/C or CC genotype (Fig. 4).

\section{Discussion}

Research is increasingly indicating the importance of glial perturbations; a crucial and often overlooked factor in SCZ ${ }^{[17]}$. Considerable evidence suggests that a range of changes in astrocyte structure and function are associated with neuropsychiatric diseases, including major depressive disorder, SCZ, and addiction ${ }^{[18]}$. GFAP is considered to be a marker of astrocyte activation, with its role in SCZ being discussed. To the best of our knowledge, this is the first study to investigate the association between GFAP and SCZ in the Chinese Han population. In this study, three GFAP tag SNPs were selected and a case-control study to investigate the association between GFAP and SCZ in a Han Chinese population was performed. The data suggests that the allele or genotype frequency of rs3785891 differs significantly between patients with SCZ and healthy control individuals, and that this SNP may be associated with SCZ.

Haplotypes are widely accepted as more specific risk markers than single alleles.Consequently, the use of haplotypes can reduce the falsepositive association in common psychiatric disorders. There was no significant correlation between different haplotypes and the risk of SCZ in this study. Increased expression profiles of cortical astrocytes have been observed in SCZ ${ }^{[19]}$. However, results from this study show that GFAP expression is not altered in the hippocampus, prefrontal cortex or stratum of SCZ.

It has been shown that SCZ is associated with altered structure and function of the OCTX region ${ }^{[20]}$. Varied evidence has found that the HIPP and its interactions with other brain regions plays a crucial role in the pathogenesis of $S C Z^{[21,22]}$. It has also been suggested that THAC and WHMT play important roles in SCZ ${ }^{[23,24]}$. Magnetic resonance imaging showed changes in the FCTX region in patients with $\mathrm{SCZ}^{[25]}$. To detect differential rs3785891 expression in the brain, eQTL analysis was performed. The results obtained from this study indicate that the expression of rs3785891 in the WHMT, THAC, HIPP, OCTX and FCTX regions appears to be higher in individuals with the T/T genotype compared to those with the $\mathrm{T} / \mathrm{C}$ or $\mathrm{C} / \mathrm{C}$ genotype. In addition to the results of the present study supporting previous research findings describing abnormalities in the WHMT, THAC, HIPP, OCTX and FCTX regions of SCZ patients, this study indicates that the genotype frequency of rs3785891 is significantly associated with the risk of SCZ in the Han Chinese population. In summary, the comprehensive analysis performed in this study indicates that GFAP may regulate the structure and function of brain regions related to SCZ by changing gene expression, thus inducing SCZ pathogenesis. However, the effect of GFAP on gene expression in the Han Chinese population and its relationship with SCZ needs to be verified in a larger cohort study. 
The advantage of this study is that original data was combined with data included in relevant databases to conduct a comprehensive analysis of the relationship between GFAP and SCZ for the first time. However, this study has several limitations that should be considered. Firstly, this is an exploratory study of the general Chinese Han population. The modest sample size makes it difficult to present any definitive statements about the association of GFAP with schizophrenia in the Han population. Secondly, the samples used in this study are from eastern China and may not represent the general situation of the Han population or other closely related populations in the region. Thirdly, we did not investigate the symptomatology, pharmacotherapy, and cognitive function of the patients in our samples. Future studies will therefore involve larger SCZ patient cohorts and focus more on functional polymorphisms and their relationship with disease state.

\section{Conclusion}

In summary, this study found that the GFAP rs3785891 polymorphism may be associated with schizophrenia in the Han Chinese population. However, due to the limited sample size, the discovery is still in its early stages. To increase confidence in these results, more genetic studies and larger sample sizes are needed in combination with a meta-analysis across different studies in different ethnic populations. The data generated from this study can serve as a reference for further research.

\section{Abbreviations}

GFAP

Glial fibrillary acidic protein

SCZ

Schizophrenia

SCZB

Schizophrenia database

SNP

Single nucleotide polymorphisms

eQTL

Expression quantitative trait loci

MAF

Minor allele frequency

HCB

Han Chinese population of Beijing

MEDU

Inferior olivary nucleus

WHMT

Intralobular white matter

SNIG

Substantia nigra

THAL

Thalamus

HIPP

Hippocampus

PUTM

Putamen

OCTX

Occipital cortex

TCTX

temporal cortex

CRBL

cerebellar cortex

OR

Odds ratio

HWE

Hardy Weinberg equilibrium

LD 
linkage disequilibrium

\section{Declarations}

Availability of data and materials

The datasets used and/or analysed during this study are available from the corresponding author on reasonable request.

\section{Acknowledgements}

Not applicable.

\section{Authors'contributions}

Wenjuan Yu and Sidi He wrote the manuscript,Yimin Yu,Shen He, Qingqing Xu and Shunying Yu were involved in the study.Jingjing Huang and Huafang Li are the corresponding authors of this article and directed the study.

\section{Funding}

This study was supported by the National Science and Technology Major Project for IND (investigational new drug)(2018ZX09734005)Clinical Research Center, Shanghai Jiao Tong University School of Medicine(DLY201620)『the strategic Priority Research Program of Chinese Academy of Sciences(XDA12040105).

\section{Author information}

\section{Affiliations}

1Shanghai mental health center, Shanghai Jiao Tong university school of medicine.

2 Shanghai key laboratory of psychotic disorders.

3 Clinical research center, Shanghai Jiao Tong university school of medicine.

\section{Corresponding author}

Correspondence to Huafang Li and Jingjing Huang.

\section{Ethics declarations}

This study was approved by the Shanghai mental health center and all participants provided voluntary written informed consent.

\section{Consent for publication}

Not Applicable.

\section{Competing interests}

The authors declare that they have no competing interests.

\section{References}

[1] Sullivan PF, Kendler KS, Neale MC. Schizophrenia as a complex trait: evidence from a meta-analysis of twin studies[J]. Arch Gen Psychiatry, 2003, 60(12): 1187-1192.

[2] Braun K, Antemano R, Helmeke C, Buchner M, Poeggel G. Juvenile separation stress induces rapid region- and layer-specific changes in S100ss- and glial fibrillary acidic protein-immunoreactivity in astrocytes of the rodent medial prefrontal cortex[J]. Neuroscience, 2009, 160(3): 629-638.

[3] Johnston-Wilson NL, Sims CD, Hofmann JP, Anderson L, Shore AD, Torrey EF, Yolken RH. Disease-specific alterations in frontal cortex brain proteins in schizophrenia, bipolar disorder, and major depressive disorder. The Stanley Neuropathology Consortium[J]. Mol Psychiatry, 2000, 5(2): 142-149. 
[4] Hol EM, Pekny M. Glial fibrillary acidic protein (GFAP) and the astrocyte intermediate filament system in diseases of the central nervous system[J]. Curr Opin Cell Biol, 2015, 32: 121-130.

[5] Miller SJ. Astrocyte Heterogeneity in the Adult Central Nervous System[J]. Front Cell Neurosci, $2018,12: 401$.

[6] Dallerac G, Rouach N. Astrocytes as new targets to improve cognitive functions[J]. Prog Neurobiol, 2016, $144: 48-67$.

[7] Blanco-Suarez E, Caldwell AL, Allen NJ. Role of astrocyte-synapse interactions in CNS disorders[J]. J Physiol, 2017, 595(6): 19031916.

[8] Williams MR, Hampton T, Pearce RK, Hirsch SR, Ansorge O, Thom M, Maier M. Astrocyte decrease in the subgenual cingulate and callosal genu in schizophrenia[J]. Eur Arch Psychiatry Clin Neurosci, 2013, 263(1): 41-52.

[9] Limoa E, Hashioka S, Miyaoka T, Tsuchie K, Arauchi R, Azis IA, Wake R, Hayashida M, Araki T, Furuya M, Liaury K, Tanra AJ, Horiguchi J. Electroconvulsive shock attenuated microgliosis and astrogliosis in the hippocampus and ameliorated schizophrenia-like behavior of Gunn rat[J]. J Neuroinflammation, 2016, 13(1): 230.

[10] Sun L, Min L, Zhou H, Li M, Shao F, Wang W. Adolescent social isolation affects schizophrenia-like behavior and astrocyte biomarkers in the PFC of adult rats[J]. Behav Brain Res, 2017, 333: 258-266.

[11] Fornito A, Bullmore ET. Reconciling abnormalities of brain network structure and function in schizophrenia[J]. Curr Opin Neurobiol, 2015, 30: 44-50.

[12] Wu Y, Yao YG, Luo XJ. SZDB: A Database for Schizophrenia Genetic Research[J]. Schizophr Bull, $2017,43(2): 459-471$.

[13] Yoshimine T, Maruno M, Kumura E, Ghulam Muhammad AK, Ninomiya H, Kato A, Hayakawa T. Stochastic determination of the chromosomal region responsible for expression of human glial fibrillary acidic protein in astrocytic tumors[J]. Neurosci Lett, 1998, 247(1): 2932.

[14] Ramasamy A, Trabzuni D, Guelfi S, Varghese V, Smith C, Walker R, De T, Coin L, de Silva R, Cookson MR, Singleton AB, Hardy J, Ryten $\mathrm{M}$, Weale ME. Genetic variability in the regulation of gene expression in ten regions of the human brain[J]. Nat Neurosci, 2014, 17(10): 14181428.

[15] Shi YY, He L. SHEsis, a powerful software platform for analyses of linkage disequilibrium, haplotype construction, and genetic association at polymorphism loci[J]. Cell Res, 2005, 15(2): 97-98.

[16] Barrett JC, Fry B, Maller J, Daly MJ. Haploview: analysis and visualization of LD and haplotype maps[J]. Bioinformatics, 2005, 21(2): 263-265.

[17] Catts VS, Wong J, Fillman SG, Fung SJ, Shannon Weickert C. Increased expression of astrocyte markers in schizophrenia: Association with neuroinflammation[J]. Aust N Z J Psychiatry, 2014, 48(8): 722-734.

[18] Kim R, Healey KL, Sepulveda-Orengo MT, Reissner KJ. Astroglial correlates of neuropsychiatric disease: From astrocytopathy to astrogliosis[J]. Prog Neuropsychopharmacol Biol Psychiatry, 2018, 87(Pt A): 126-146.

[19] Toker L, Mancarci BO, Tripathy S, Pavlidis P. Transcriptomic Evidence for Alterations in Astrocytes and Parvalbumin Interneurons in Subjects With Bipolar Disorder and Schizophrenia[J]. Biol Psychiatry, 2018, 84(11): 787-796.

[20] Tohid H, Faizan M, Faizan U. Alterations of the occipital lobe in schizophrenia[J]. Neurosciences (Riyadh), 2015, 20(3): $213-224$.

[21] Tamminga CA, Stan AD, Wagner AD. The hippocampal formation in schizophrenia[J]. Am J Psychiatry, 2010, 167(10): 1178-1193.

[22] Freedman R, Goldowitz D. Studies on the hippocampal formation: From basic development to clinical applications: Studies on schizophrenia[J]. Prog Neurobiol, 2010, 90(2): 263-275.

[23] Pergola G, Selvaggi P, Trizio S, Bertolino A, Blasi G. The role of the thalamus in schizophrenia from a neuroimaging perspective[J]. Neurosci Biobehav Rev, 2015, 54: 57-75.

[24] Najjar S, Pearlman DM. Neuroinflammation and white matter pathology in schizophrenia: systematic review[J]. Schizophr Res, 2015, 161(1): 102-112. 
[25] Vostrikov VM, Uranova NA. [Deficit of oligodendrocytes in the frontal cortex in schizophrenia][J]. Zh Nevrol Psikhiatr Im S S Korsakova, 2018, 118(5): 100-103.

\section{Tables}

\begin{tabular}{|c|c|c|c|c|c|c|c|c|c|c|c|c|}
\hline & $\mathrm{N}$ & $\begin{array}{l}\text { Allele } \\
\mathrm{N}(\%)\end{array}$ & & $X^{2}$ & $p$ & MAF(\%) & $\begin{array}{l}\text { Genotype } \\
\mathrm{n}(\%)\end{array}$ & & & $\mathrm{X}^{2}$ & $P$ & $\begin{array}{l}P \text { of } \\
\text { HWE }\end{array}$ \\
\hline rs2289681 & & C & $\mathrm{T}$ & & & $\mathrm{T}$ & $\mathrm{CC}$ & CT & TT & & & \\
\hline case & 629 & 1049(83.4) & $209(16.6)$ & 0.32 & 0.57 & 14.1 & $437(69.5)$ & $175(27.8)$ & $17(2.7)$ & 0.37 & 0.83 & 0.81 \\
\hline Control & 649 & 1093(84.2) & 205(15.8) & & & & 461(71.0) & $171(26.3)$ & $17(2.6)$ & & & \\
\hline rs3785891 & & C & $\mathrm{T}$ & & & $\mathrm{T}$ & $\mathrm{CC}$ & CT & TT & & & \\
\hline case & 628 & $954(76.0)$ & $302(24.0)$ & 4.17 & 0.04 & 26.0 & $360(57.3)$ & 234(37.3) & $34(5.4)$ & 4.54 & 0.10 & 0.21 \\
\hline control & 599 & $940(72.4)$ & $358(27.6)$ & & & & $334(51.5)$ & 272(41.9) & $43(6.6)$ & & & \\
\hline rs3744469 & & C & G & & & C & $\mathrm{CC}$ & CG & GG & & & \\
\hline case & 629 & $39(3.1)$ & $1219(96.9)$ & 1.03 & 0.23 & 5.3 & $0(0.0)$ & $39(6.2)$ & $590(93.8)$ & 2.33 & 0.31 & 0.27 \\
\hline control & 652 & $50(3.8)$ & $1254(96.2)$ & & & & $2(0.3)$ & $46(7.1)$ & $604(92.6)$ & & & \\
\hline
\end{tabular}

Table 1. The allele and genotype distributions of the GFAP tag SNPs in SCZ and healthy controls

\begin{tabular}{|llllll|}
\hline Block & Haplotype & Frequency in case & Frequency in control & $X^{2}$ & $P$ \\
\hline rs2289681-rs3785891 & CC & 0.590 & 0.567 & 1.455 & 0.2278 \\
\cline { 2 - 6 } & CT & 0.247 & 0.276 & 2.79 & 0.0949 \\
\hline & TC & 0.163 & 0.158 & 0.141 & 0.7069 \\
\hline
\end{tabular}

Table 2. GFAP haplotype frequency analysis of between SCZ and healthy control participants

\section{Figures}

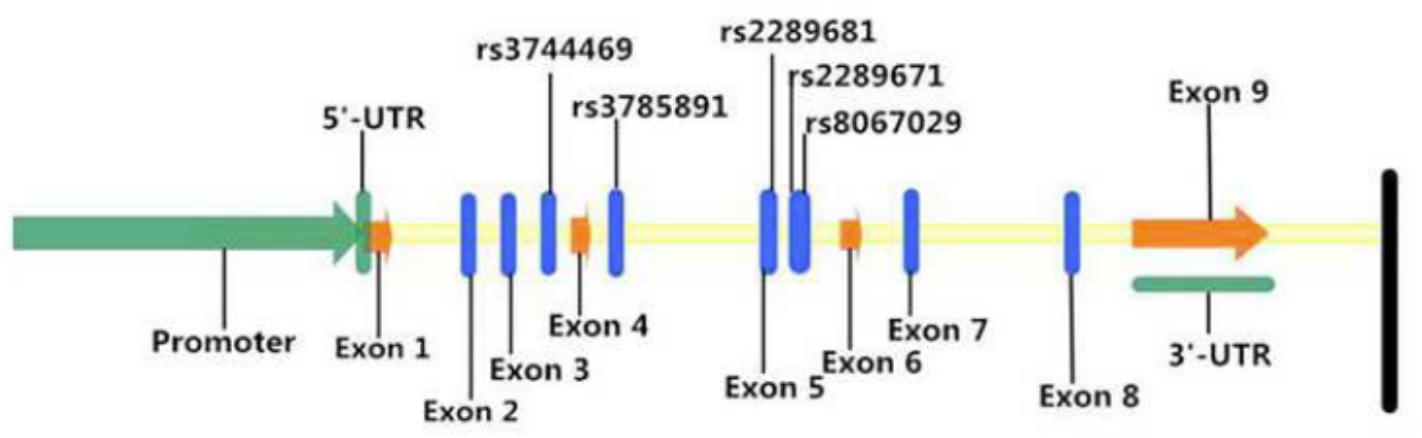

\section{Figure 1}

The structure of GFAP and the selected tag SNPs 


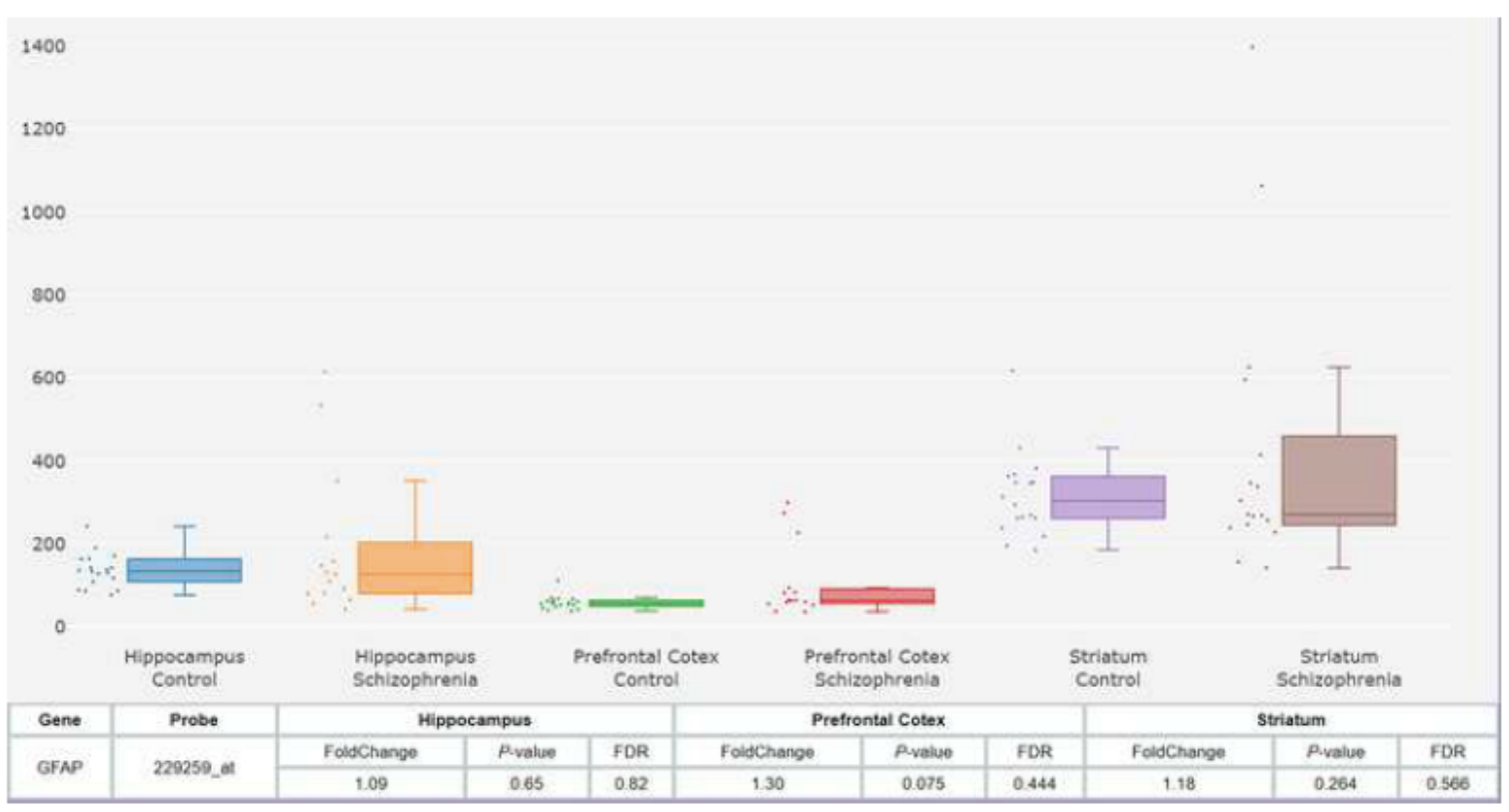

\section{Figure 2}

Differential expression of GFAP in brain regions between patients with SCZ and healthy controls. Each bar represents the average GFAP expression level.Error bars represent the standard deviation of the mean.Data was extracted from the SCZB(http://www.szdb.org/).

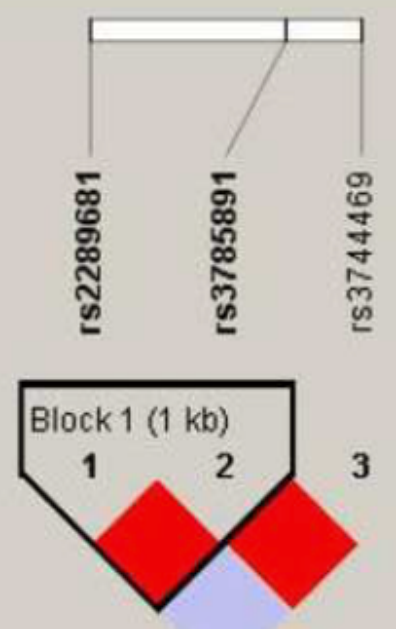

The linkage disequilibrium of the tag SNPs of GFAP.

\section{Figure 3}

The linkage disequilibrium of the GFAP tag SNPs 


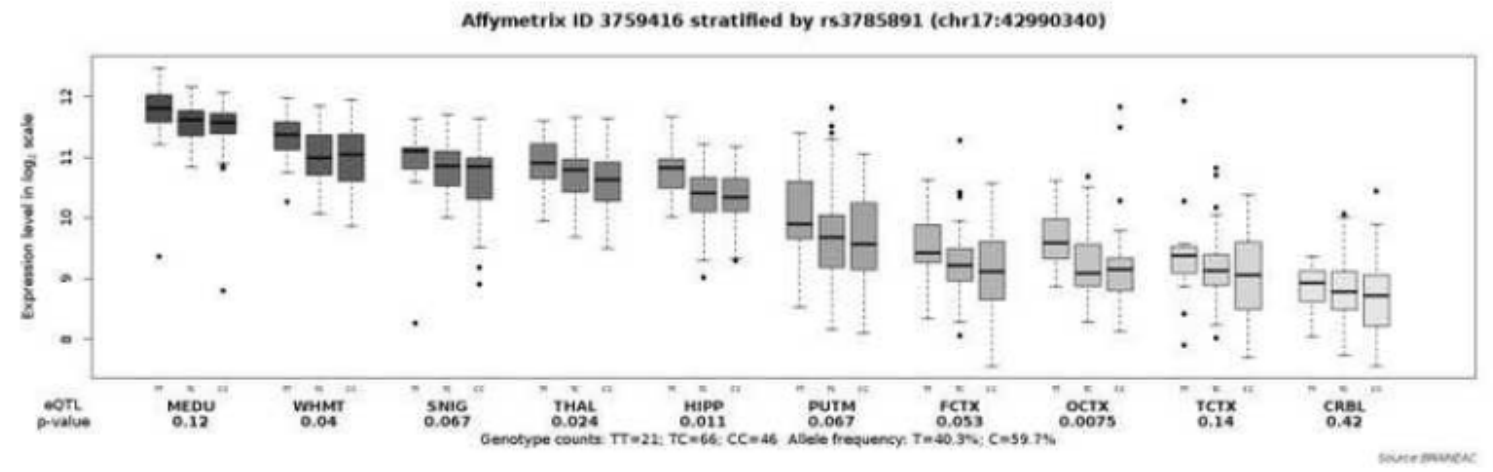

\section{Figure 4}

Association of rs3785891 with GFAP expression levels in 10 brain regions (Affymetrix ID t3759416). Data were extracted from the BRAINEAC database (http://peana-od.inf.um.es:8080/UKBECv12/).Abbreviations: BRAINEAC, The Brain eQTL Almanac;GFAP, Glial fibrillary acidic protein; eQTL, expression quantitative trait locus; MEDU, inferior olivary nucleus; WHMT, intralobular white matter; SNIG, substantia nigra; THAL, thalamus; HIPP, hippocampus; PUTM, putamen; OCTX, occipital cortex; TCTX, temporal cortex; CRBL, cerebellar cortex. 